\title{
Searches for rare and forbidden kaon decays at the NA62 experiment at CERN
}

\author{
Gianluca Lamanna*† \\ Universita \& INFN Pisa, Italy \\ E-mail: gianluca.lamanna@cern.ch
}

\begin{abstract}
The NA62 experiment at the CERN SPS aims at measuring the branching ratio (BR) of the rare $K^{+} \rightarrow \pi^{+} v \bar{v}$ decay, with a precision of $\sim 10 \%$. This goal will be achieved after two years of data taking by collecting $\sim 10^{13} K^{+}$decays in the fiducial volume. The $K^{+} \rightarrow \pi^{+} v \bar{v}$ is a "golden mode" in flavor physics because of the precise theoretical prediction. Thanks to the unprecedent kaon flux, it will also be possible to search for many other forbidden processes, including leptor flavor violation modes, sterile neutrinos, supersymmetric particles. The expected NA62 performances will wallow the exclusion limits for several decay modes to be improved. The experiment will start collecting data in late 2014.
\end{abstract}

The European Physical Society Conference on High Energy Physics

18-24 July, 2013

Stockholm, Sweden

\footnotetext{
* Speaker.

†for the NA62 Collaboration: G. Aglieri Rinella, F. Ambrosino, B. Angelucci, A. Antonelli, G. Anzivino, R. Arcidiacono, I. Azhinenko, S. Balev, A. Biagioni, C. Biino, A. Bizzeti, T. Blazek, A. Blik, B. Bloch-Devaux, V. Bolotov, V. Bonaiuto, D. Britton, G. Britvich, N. Brook, F. Bucci, V. Buescher, F. Butin, T. Capussela, V. Carassiti, N. Cartiglia, A. Cassese, A. Catinaccio, A. Ceccucci, P. Cenci, V. Cerny, C. Cerri, O. Chikilev, R. Ciaranfi, G. Collazuol, P. Cooke, P. Cooper, E. Cortina Gil, F. Costantini, A. Cotta Ramusino, D. Coward, G. D’Agostini, J. Dainton, P. Dalpiaz, H. Danielsson, N. De Simone, D. Di Filippo, L. Di Lella, N. Doble, V. Duk, V. Elsha, J. Engelfried, V. Falaleev, R. Fantechi, L. Federici, M. Fiorini, J. Fry, A. Fucci, S. Gallorini, L. Gatignon, A. Gianoli, S. Giudici, L. Glonti, F. Gonnella, E. Goudzovski, R. Guida, E. Gushchin, F. Hahn, B. Hallgren, H. Heath, F. Herman, E. Iacopini, O. Jamet, P. Jarron, K. Kampf, J. Kaplon, V. Karjavin, V. Kekelidze, A. Khudyakov, Yu. Kiryushin, K. Kleinknecht, A. Kluge, M. Koval, V. Kozhuharov, M. Krivda, J. Kunze, G. Lamanna, C. Lazzeroni, R. Leitner, M. Lenti, E. Leonardi, P. Lichard, R. Lietava, L. Litov, D. Lomidze, A. Lonardo, N. Lurkin, D. Madigozhin, G. Maire, A. Makarov, I. Mannelli, G. Mannocchi, A. Mapelli, F. Marchetto, P. Massarotti, K. Massri, P. Matak, G. Mazza, E. Menichetti, M. Mirra, M. Misheva, N. Molokanova, M. Morel, M. Moulson, S. Movchan, D. Munday, M. Napolitano, F. Newson, A. Norton, M. Noy, G. Nuessle, V. Obraztsov, S. Padolski, R. Page, T. Pak, V. Palladino, A. Pardons, E. Pedreschi, M. Pepe, F. Petrucci, R. Piandani, M. Piccini, J. Pinzino, M. Pivanti, I. Polenkevich, I. Popov, Yu. Potrebenikov, D. Protopopescu, F. Raffaelli, M. Raggi, P. Riedler, A. Romano, P. Rubin, G. Ruggiero, V. Ryjov, A. Salamon, G. Salina, V. Samsonov, E. Santovetti, G. Saracino, F. Sargeni, S. Schifano, V. Semenov, A. Sergi, M. Serra, S. Shkarovskiy, A. Sotnikov, V. Sougonyaev, M. Sozzi, T. Spadaro, F. Spinella, R. Staley, M. Statera, P. Sutcliffe, N. Szilasi, M. Valdata-Nappi, P. Valente, B. Velghe, M. Veltri, S. Venditti, M. Vormstein, H. Wahl, R. Wanke, P. Wertelaers, A. Winhart, R. Winston, B. Wrona, O. Yushchenko, M. Zamkovsky, A. Zinchenko.
} 


\section{Introduction}

The kaon system, in spite of its relative simplicity (relatively few decay channels, low finalstate multiplicities), offers several opportunities to study deeply the Standard Model (SM). On one side the processes of flavor changing neutral current (FCNC) are a unique laboratory for studying flavor dynamics as described by the CKM matrix and possible extensions, on the other hand processes that violate lepton flavor conservation are particularly sensitive to the effects of new physics. The FCNC decays (for instance $K_{L} \rightarrow \mu^{+} \mu^{-}$and $K \rightarrow \pi l^{+} l^{-}$) are highly suppressed in the SM and proceed normally only through second order loop diagrams (boxes and penguins). Among the flavor changing neutral current $\mathrm{K}$ and $\mathrm{B}$ decays, the $K \rightarrow \pi v \bar{v}$ decays play a key role in the search for new physics through the underlying mechanisms of flavor mixing. These decays are strongly suppressed in the SM (the highest CKM suppression), and are dominated by top-quark loop contributions. The SM branching ratios have been computed to an exceptionally high precision with respect to other loop-induced meson decays: $B R\left(K^{+} \rightarrow \pi^{+} v \bar{v}\right)=(7.81 \pm 0.75 \pm 0.29) \times 10^{-11}$ and $B R\left(K^{0} \rightarrow \pi^{0} v \bar{v}\right)=(2.43 \pm 0.39 \pm 0.06) \times 10^{-11}$, the uncertainties are dominated by parametric ones, and the irreducible theoretical uncertainties are at $1 \%$ level [1]. The extreme theoretical cleanliness of these decays remains also in certain new physics scenarios.

Experimentally, the $K^{+} \rightarrow \pi^{+} v \bar{v}$ decay has been observed by the BNL E787/E949 experiments, with a branching ratio of $1.73_{-1.05}^{+1.15} \times 10^{-10}$ [2], based on 7 candidates. The achieved precision is inferior to that of the SM expectation. Because of the excellent precision in the theoretical prediction even relatively small difference with respect to the SM could be a clear signal of new physics. The simultaneous measurement in the charged and neutral kaons highly constraints alternative models of physics beyond the SM [3]. In addition the precise measurement of $K^{+} \rightarrow \pi^{+} v \bar{v}$ will be an alternative measurement of $V_{t d}$ with respect to the B decays.

The lepton flavor conservation (LF) is an accidental symmetry of the SM, in the sense that there is no a fundamental reason prevents its possible violation. The observation of a small LF violation in neutrino mixing leads to a very small prediction in the SM for the BR of Lepton Flavor Violation (LFV) modes in meson decays. For this reason any observation of LFV processes is a clear signal of physics beyond the SM. Several extensions of SM can accommodate the LFV including supersymmetry, extra dimensions and others. Thanks to the availability of high intensity kaon beams and the clear experimental signature with respect to background processes, the LFV search in kaon sector have reached upper limits to the BR at level of $10^{-10}$. In table 1 we summarize the present status for the main LFV decay channels in charged kaon decays. Together with FCNC

\begin{tabular}{llll}
\hline Process & Upper Limit (90\% CL) & Experiment & Ref. \\
\hline$K^{+} \rightarrow \pi^{+} \mu^{+} e^{-}$ & $1.3 \times 10^{-11}$ & BNL 777/865 & {$[4]$} \\
$K^{+} \rightarrow \pi^{+} \mu^{-} e^{+}$ & $5.2 \times 10^{-10}$ & BNL 865 & {$[5]$} \\
$K^{+} \rightarrow \pi^{-} \mu^{+} e^{+}$ & $5.0 \times 10^{-10}$ & BNL 865 & {$[5]$} \\
$K^{+} \rightarrow \pi^{-} e^{+} e^{+}$ & $6.4 \times 10^{-10}$ & BNL 865 & {$[5]$} \\
$K^{+} \rightarrow \pi^{-} \mu^{+} \mu^{+}$ & $1.1 \times 10^{-9}$ & NA48/2 & {$[6]$} \\
$K^{+} \rightarrow \mu^{-} v e^{+} e^{+}$ & $2.0 \times 10^{-8}$ & Geneva-Saclay & {$[7]$}
\end{tabular}

Table 1: Current status of searches for the main LFV modes in Kaon decays. 


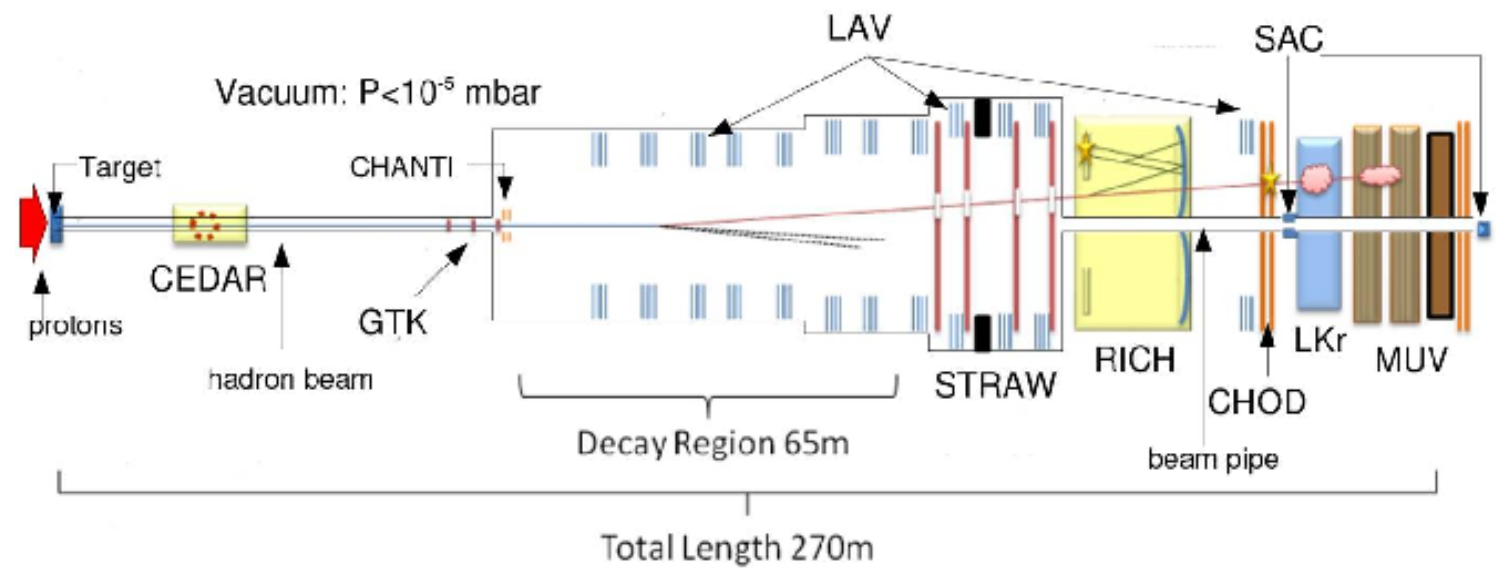

Figure 1: Layout of the NA62 experiment at CERN.

and LFV modes, the kaon sector allows to search for other forbidden processes and exotic particles, such dark photons and sterile neutrinos, as well as angular momentum violation and others.

The NA62 experiment at the CERN SPS will start in late 2014 to study ultrarare kaon decays. It will observe $\sim 10^{13} K^{+}$decays in its fiducial volume to carry out a rich program to search for $K^{+}$decays, including the measurement of BR for the decay $K^{+} \rightarrow \pi^{+} v \bar{v}$ and many others LFV and SM forbidden decays as well as the search for new particles, as described in this paper.

\section{The NA62 experiment}

The layout of the NA62 experiment is shown in fig.1 . A high intensity and high energy kaon beam is exploited to provide kaon decays in flight over a long decay region. The unseparated hadron beam is obtained by the primary $400 \mathrm{GeV} / c$ proton beam from the SPS accelerator impinging on a beryllium target. About $\sim 6 \%$ of the total amount of particles in the secondary $75 \mathrm{GeV} / \mathrm{c}$ beam are kaons (the rest are mainly pions $(\sim 70 \%)$, protons $(\sim 23 \%)$ and positrons $(\sim 1 \%$ after filtering through a $\mathrm{W}$ foil)). The identification of the kaons in the beam is done in a differential hydrogen Cerenkov counter (CEDAR). A silicon pixel beam spectrometer (Gigatracker) placed on the $750 \mathrm{MHz}$ hadron beam, measures the momentum and the direction of the particles improving the resolution on the beam momentum from $1 \%$ to $0.2 \%$ (RMS). The decay region is housed in $\mathrm{a} \sim 80 \mathrm{~m}$ long $\sim 2.5 \mathrm{~m}$ diameter evacuated tube $\left(10^{-6} \mathrm{mbar}\right)$. The charged particles produced in kaon decays (pions, muons and electrons) are measured by a straw tubes spectrometer (STRAW), composed by four stations and an analyzing magnet integrated directly inside the evacuated decay region. The Straw spectrometer is very thin $\left(<0.5 X_{0}\right.$ per chamber $)$ to minimize the interactions with the photons coming from the kaon decays. A liquid krypton electromagnetic calorimeter ( $\mathrm{LKr}$ ) is devoted to measure photons and electrons in the forward direction, while a complex of 12 rings of lead glass blocks(LAVs) are placed along the decay region to identify the large angle photons. A $1 \mathrm{~atm}$ Neon RICH is used to distinguish among muons and pions in the $15-35 \mathrm{GeV} / \mathrm{c}$ range, increasing the particle identification power of the muon identification system (MUVs) placed just beyond the LKr. 


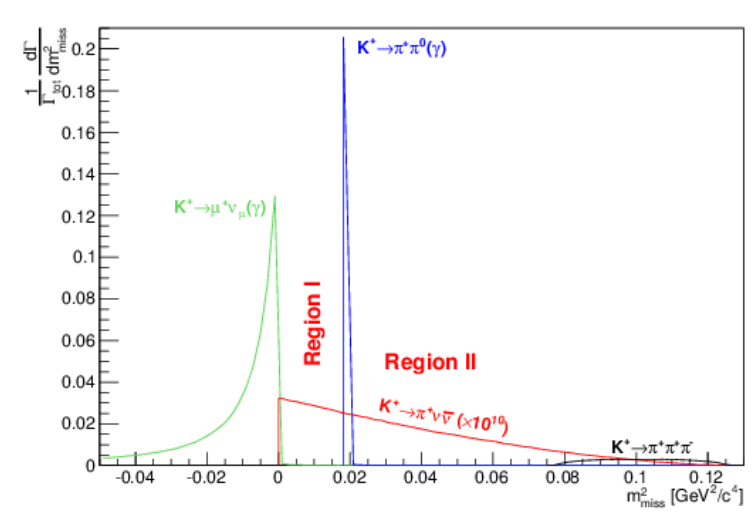

Figure 2: Definition of signal region in $m_{\text {miss }}^{2}$ distribution. The backgrounds are normalized according to their branching ratio; the signal is multiplied by a factor $10^{10}$.

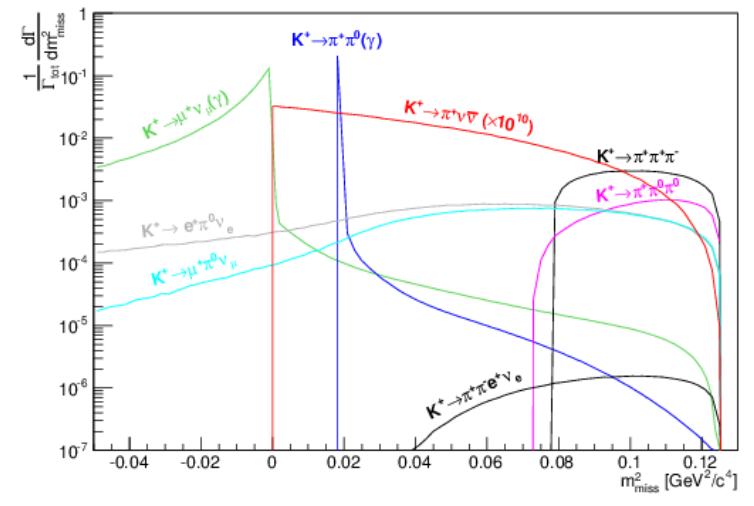

Figure 3: $m_{m i s s}^{2}$ distribution for signal and backgrounds from the main $K^{+}$decay modes. The backgrounds are normalized according to their branching ratio; the signal is multiplied by a factor $10^{10}$.

At nominal intensity the main detectors will be exposed at a rate of $10 \mathrm{MHz}$ of events. A multilevel trigger system is designed to reduce such intensity at few $\mathrm{kHz}$. The first level (L0) is built in hardware by using the primitives produced in FPGA in the readout boards (TEL62), and reduces the rate from $10 \mathrm{MHz}$ to $1 \mathrm{MHz}$. The additional reduction (at least a factor of 100) is achieved in the software levels (L1 and L2) running in the online PC farm. While the L0 latency is limited to $1 \mathrm{~ms}$, the latency in the software levels is in the order of few seconds.

\section{3. $K^{+} \rightarrow \pi^{+} v \bar{v}$}

The main goal of the NA62 experiment is the measurement of the $K^{+} \rightarrow \pi v \bar{v}$ decay rate at the $10 \%$ precision level, which would constitute a significant test of the SM. The experiment is expected to collect about 100 signal events in two years of data taking, keeping the systematic uncertainties and backgrounds as low as possible. Assuming 10\% signal acceptance and the SM decay rate, the necessary kaon flux corresponds to, at least, $10^{13} \mathrm{~K}^{+}$decays in the fiducial volume. In order to achieve a small systematic uncertainty, a rejection factor for generic kaon decays of the order of $10^{12}$ is required, and the background suppression factors need to be measured directly from the data. The signature of the signal is one track in the final state matched with one $K^{+}$track in the beam. The $m_{\text {miss }}^{2}=\left(P_{K}-P_{\pi}\right)^{2}$, with $P_{K}$ and $P_{\pi}^{+}$the four momenta of the $K^{+}$and the charged pion, fully describes the kinematics of the decay. Backgrounds come from all the $K^{+}$decay modes and from accidental single tracks matched with a $K^{+}$-like track. The $m_{\text {miss }}^{2}$ allows to define two signal regions, before and after the $\pi^{0}$ peak, as shown in fig.2.

Anyway the background in these regions is still orders of magnitude larger than the signal, due to resolution effects, radiative tails, accidental particles and rare decays as shown in fig.3. Additional rejection factor is provided by veto and particle identification system. The signal events will be selected with a single track in Straw spectrometer in time with a kaon identified in the CEDAR and measured in the GTK. The kaon identification allows to suppress most of the background due 


\begin{tabular}{ll}
\hline Decay & Events/year \\
\hline$K^{+} \rightarrow \pi^{+} \pi^{0}$ & 5 \\
$K^{+} \rightarrow \pi^{+} \pi^{+} \pi^{-}$ & 1 \\
$K^{+} \rightarrow \pi^{+} \pi^{-} e+v$ & $<1$ \\
$K^{+} \rightarrow \pi^{+} \pi^{0} \gamma(I B)$ & $<1$ \\
$K^{+} \rightarrow \mu^{+} v \gamma$ & 1.5 \\
other rare decays & 0.5 \\
\hline Total Background & $<10$ \\
\hline$K^{+} \rightarrow \pi^{+} v \bar{v}$ & 45 \\
\hline
\end{tabular}

Table 2: Signal and background from $K^{+}$decays estimated from the sensitivity studies.

\begin{tabular}{ll}
\hline Process & Acceptance \\
\hline$K^{+} \rightarrow \pi^{+} \mu^{+} e^{-}$ & $\sim 10 \%$ \\
$K^{+} \rightarrow \pi^{+} \mu^{-} e^{+}$ & $\sim 10 \%$ \\
$K^{+} \rightarrow \pi^{-} \mu^{+} e^{+}$ & $\sim 10 \%$ \\
$K^{+} \rightarrow \pi^{-} e^{+} e^{+}$ & $\sim 5 \%$ \\
$K^{+} \rightarrow \pi^{-} \mu^{+} \mu^{+}$ & $\sim 20 \%$ \\
$K^{+} \rightarrow \mu^{-} v e^{+} e^{+}$ & $\sim 2 \%$ \\
$\pi^{0} \rightarrow \mu^{-} e^{+}$ & $\sim 2 \%$ \\
$\pi^{0} \rightarrow \mu^{+} e^{-}$ & $\sim 2 \%$ \\
$\pi^{+} \rightarrow \mu^{-} e^{+} e^{+} v$ & $\sim 2 \%$ \\
\hline
\end{tabular}

Table 3: Estimated acceptance for $K^{+}$and $\pi^{0}$ LFV modes.

to the interactions of the beam with residual gas in the decay region. The pion identification will be done by using the RICH and the calorimeters ( $\mathrm{LKr}$ and first two stations of the MUV system). Events with signals in the LAV and other veto systems compatible with a $\gamma$ hypothesis will be rejected. Finally two global requirements will be applied: the kaon decay has to take place in the first $60 \mathrm{~m}$ of the decay volume; the measured momentum of the downstream $\pi^{+}$must be between 15 and $35 \mathrm{GeV} / c$.

In table 2 the numbers of signal and background events are estimated from sensitivity studies. The numbers are normalized to $\sim 4.5 \times 10^{12}$ kaon decays per year.

\section{4. "Exotics" and "Forbiddens"}

The high intensity of the kaon flux available for the NA62 experiment combined with a flexible trigger system allow to carry out a rich program of kaon and $\pi^{0}$ decays forbidden in the SM with unprecedented precision. In table 3 we summarize the most interesting decays modes, with a preliminary estimate of the acceptance (including a rough estimation of trigger efficiency) in the NA62 experiment. The $K^{+} \rightarrow \pi^{-} \mu^{+} \mu^{+}$is particularly interesting from theoretical point of view. As shown in fig.4, lepton flavor violation implies the exchange of a virtual Majorana neutrino [8]. This process is similar to the neutrino-less nuclear double beta decay, but, in this case, the second generation is involved. A dedicated trigger will be designed to collect LFV events, eventually by using online computing on GPU [?]. The present limit to the BR is given by the NA48/2 experiment [6]. NA62 aims at improving the upper limit at least at level of $\sim 10^{-12}$.

In addition to LFV modes other searches of exotics particles will be carried out by the NA62 experiment. The existence of low mass boson is requested by several theories to explain some of the SM anomalies (excess of positrons in cosmic rays [10], Dama/Libra dark matter signal [11] , $(g-2)_{\mu}$ anomaly [12]). This new particle, called either Dark Photon or U boson, can be seen in the $\pi^{0}$ decays, for instance, by studying the $\pi^{0} \rightarrow U \gamma$, with $U \rightarrow e^{+} e^{-}$, decay. More than $20 \%$ of total BR have neutral pions in the final state. In two years of data taking the NA62 detectors will be exposed to $\sim 2.5 \times 10^{12} \pi^{0}$ decays. The prospect is to improve the exclusion limit in the mass range for the dark photon between tens of $\mathrm{MeV} / c^{2}$ and $100 \mathrm{MeV} / c^{2}$. Several other searches will 


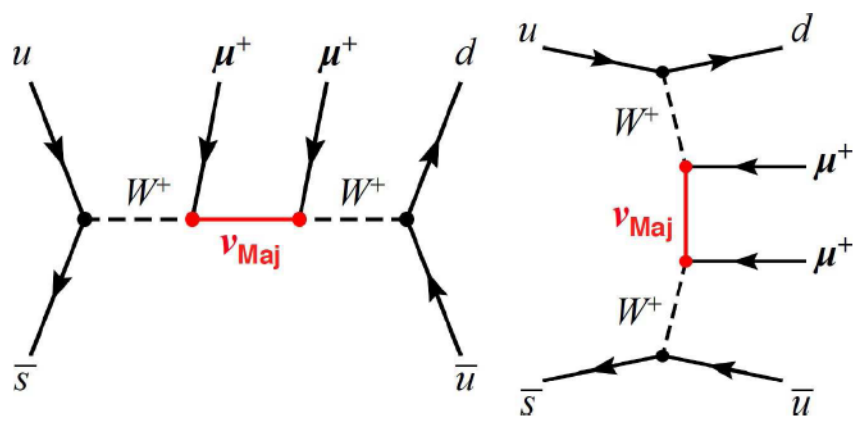

Figure 4: Lowest-order diagrams for $K^{+} \rightarrow \pi^{-} \mu^{+} \mu^{+}$.

be carried out by exploiting the clean environment offered by the kaon system, for example: search for light sgoldstino (in $K^{+} \rightarrow \pi^{+} \pi^{0} S$ ), C violation in electromagnetic interaction (in $\pi^{0} \rightarrow \gamma \gamma \gamma$ ), right-handed sterile neutrinos (in $\pi^{0} \rightarrow v \bar{v}$ and $K^{+} \rightarrow \mu v$ ), angular momentum conservation (in $\left.K^{+} \rightarrow \pi^{+} \gamma\right)$ and many others.

\section{Conclusions}

The NA62 experiment at the CERN SPS will start collecting data in 2014. A huge flux of kaons will be exploited to study ultrarare kaon decays. The main goal of the experiment will be the measurement of BR of $K^{+} \rightarrow \pi^{+} v \bar{v}$ decay, precisely computed in the SM with high precision. A discrepancy at level of $\sim 10 \%$ with respect to the predicted value will be a clear signal of physics beyond the SM. With $10^{13} K^{+}$in its fiducial volume in two years of data taking, the NA62 experiment will have a single event sensitivity of $10^{-12}\left(10^{-11}\right)$ for a number of $K^{+}\left(\pi^{0}\right)$ LFV decays, as well as the potential to improve the upper limits for several searches for effects and particles not foreseen in the SM.

\section{References}

[1] J. Brod, M. Gorbahn and E. Stamou, Phys. Rev. D 83 (2011) 034030 [arXiv:1009.0947 [hep-ph]].

[2] A. V. Artamonov et al. [BNL-E949 Collaboration], Phys. Rev. D 79 (2009) 092004 [arXiv:0903.0030 [hep-ex]].

[3] M. Blanke, arXiv:1305.5671 [hep-ph].

[4] A. Sher [E865 Collaboration], Int. J. Mod. Phys. A 20 (2005) 3349.

[5] R. Appel, G. S. Atoyan, B. Bassalleck, D. R. Bergman, N. Cheung, S. Dhawan, H. Do and J. Egger et al., Phys. Rev. Lett. 85 (2000) 2877 [hep-ex/0006003].

[6] J. R. Batley et al. [NA48/2 Collaboration], Phys. Lett. B 697 (2011) 107 [arXiv:1011.4817 [hep-ex]].

[7] A. M. Diamant-Berger, P. Bloch, B. Bloch-Devaux, N. Do-Duc, G. Marel, R. Turlay, P. Extermann and J. Fischer et al., Phys. Lett. B 62 (1976) 485.

[8] A. Atre, T. Han, S. Pascoli and B. Zhang, JHEP 0905 (2009) 030 [arXiv:0901.3589 [hep-ph]].

[9] G. Collazuol, G. Lamanna, J. Pinzino and M. S. Sozzi, Nucl. Instrum. Meth. A 662 (2012) 49. 
[10] O. Adriani et al. [PAMELA Collaboration], Nature 458 (2009) 607 [arXiv:0810.4995 [astro-ph]].

[11] R. Bernabei et al. [DAMA Collaboration], Eur. Phys. J. C 56 (2008) 333 [arXiv:0804.2741 [astro-ph]].

[12] F. Jegerlehner and A. Nyffeler, Phys. Rept. 477 (2009) 1 [arXiv:0902.3360 [hep-ph]]. 